\title{
Atmospheric Wind Measurements with the High-Resolution Doppler Imager
}

\author{
Heinz J. Grassl, ${ }^{*}$ Wilbert R. Skinner, ${ }^{\dagger}$ Paul B. Hays,,${ }^{\ddagger}$ David A. Gell,${ }^{\S}$ Mark D. Burrage, ${ }^{\mathbb{I}}$ David A. Ortland,${ }^{\S}$ \\ Alan R. Marshall, ${ }^{* *}$ and Vincent J. Abreu ${ }^{\dagger \dagger}$ \\ University of Michigan, Ann Arbor, Michigan 48109-2143
}

\begin{abstract}
The high-resolution Doppler imager (HRDI) on the Upper Atmosphere Research Satellite is a remote sensing instrument used to observe winds in the Earth's stratosphere, mesosphere, and lower thermosphere. Winds are measured by determining the Doppler shift of emission (mesosphere and lower thermosphere) and absorption (stratosphere) lines of the $\mathrm{O}_{2}$ atmospheric $\left(b^{1} \Sigma_{g}^{+}-X^{3} \Sigma_{g}^{-}\right)$band. The HRDI is a triple-etalon Fabry-Perot interferometer with a resolution of $\approx 0.05 \mathrm{~cm}^{-1}$ and very good white-light rejection. Careful design and calibration has limited systematic errors in the wind determination to less than $5 \mathrm{~m} / \mathrm{s}$.
\end{abstract}

\section{Nomenclature}

$c \quad=$ speed of light, $3 \times 10^{8} \mathrm{~m} / \mathrm{s}$

$C_{i} \quad=$ signal observed on detector channel $i$

$C_{\max }=$ signal on the channel with the largest number of counts

$f(t)=$ long-term instrument drift arising from the gradual adjustment to the space environment, $(\mathrm{m} / \mathrm{s}) /$ day

$i \quad=$ channel number

$M \quad$ = order of interference

$N \quad=$ total finesse

$N_{A} \quad=$ aperture finesse

$N_{D} \quad=$ defect finesse

$N_{R} \quad=$ reflectivity finesse, $\pi \sqrt{R} /(1-R)$

$R \quad=$ reflectivity of each etalon plate

$R_{E} \quad=$ radius of the Earth, $\mathrm{m}$

$T \quad=$ mean instrument temperature, ${ }^{\circ} \mathrm{C}$

$t \quad=$ separation between the plates, $\mathrm{cm}$

$T_{E} \quad=$ rotation period of the Earth, $\mathrm{s}$

$U=$ measured line-of-sight velocity, $\mathrm{m} / \mathrm{s}$

$U_{0} \quad=$ zero wind reference position, $\mathrm{m} / \mathrm{s}$

$U_{\text {los }}=$ line-of-sight wind

$U_{s} \quad=$ spacecraft speed, $\mathrm{m} / \mathrm{s}$

$\alpha \quad=$ linear change in zero position with instrument mean temperature, $(\mathrm{m} / \mathrm{s}) /{ }^{\circ} \mathrm{C}$

$\beta \quad=$ azimuth angle of observation, referenced to spacecraft velocity vector, deg

$\Delta \bar{\nu} \quad=$ integrated instrument transmittance, $\mathrm{cm}^{-1}$

$\Delta v_{\mathrm{etl}}=$ spectral region through etalon collected by detector, $\mathrm{cm}^{-1}$

$\Delta v_{\mathrm{FSR}}=$ etalon free spectral range, $\mathrm{cm}^{-1}$

$\varepsilon \quad=$ elevation angle of the observation, referenced to spacecraft velocity vector, deg

Presented as Paper 93-0123 at the AIAA 31st Aerospace Sciences Meeting, Reno, NV, Jan. 11-14, 1993; received June, 9, 1993; revision received Aug. 29, 1994; accepted for publication Sept. 3, 1994. Copyright (C) 1994 by the American Institute of Aeronautics and Astronautics, Inc. All rights reserved.

${ }^{*}$ Senior Engineer in Research, Space Physics Research Laboratory, Department of Atmospheric, Oceanic and Space Sciences.

†Associate Research Scientist, Space Physics Research Laboratory, Department of Atmospheric, Oceanic and Space Sciences.

${ }^{\ddagger}$ Professor, Research Scientist, Space Physics Research Laboratory, Department of Atmospheric, Oceanic and Space Sciences.

${ }^{\$}$ Senior Research Associate, Space Physics Research Laboratory, Department of Atmospheric, Oceanic and Space Sciences.

IAssistant Research Scientist, Space Physics Research Laboratory, Department of Atmospheric, Oceanic and Space Sciences.

${ }^{* *}$ Program Analyst, Space Physics Research Laboratory, Department of Atmospheric, Oceanic and Space Sciences.

${ }^{\dagger \dagger}$ Research Scientist, Space Physics Research Laboratory, Department of Atmospheric, Oceanic and Space Sciences. $\theta \quad=$ angle of incidence in the gap, deg

$\mu \quad=$ index of refraction of the material between the plates

$v \quad=$ wave number of light, $\mathrm{cm}^{-1}$

$\phi \quad=$ latitude of observation, deg

$\psi \quad=$ observation bearing angle from north, deg

$\Omega_{\mathrm{etl}} \quad=$ solid angle passing through etalon, $\mathrm{sr}$

\section{Introduction}

$\mathbf{T}$ HE distribution of most chemical species in the stratosphere is affected by both dynamical and chemical processes. Conversely, the distribution of certain photochemical species, such as ozone, can influence the radiative budget of the stratosphere, affecting temperatures and motions. Until recently, satellite remote observations of the stratosphere have provided only temperature and constituent measurements. Global horizontal winds have been deduced from temperature fields by using the thermal-wind relationships, which relate the vertical shear of the geostrophic wind components to horizontal temperature gradients. These equations, however, are only a good approximation for large-scale, lowfrequency, extratropical flows. ${ }^{1}$ The high-resolution Doppler imager (HRDI) on the Upper Atmosphere Research Satellite (UARS) is providing the first direct measurement of the global horizontal wind field in the stratosphere, mesosphere, and thermosphere. The UARS was launched September 12, 1991, into a $585-\mathrm{km}$ circular orbit inclined $57 \mathrm{deg}$ to the equator. ${ }^{2}$ The spacecraft carries ten instruments designed to study the chemistry and dynamics of the stratosphere and above. Knowledge of the stratospheric wind field will help to determine how transport and mixing influence the ozone budget in the lower stratosphere, and to quantify the mean and eddy circulations associated with the observed semiannual oscillations. In the mesosphere, the wind measurements will contribute to the understanding of the relative role of turbulent diffusion and bulk advection in accounting for mesospheric tracer budgets.

The goal of the HRDI is to measure the vector winds in the stratosphere $(10-40 \mathrm{~km})$, mesosphere, and lower thermosphere $(\approx 50-120$ $\mathrm{km})$ during the day, and in the lower thermosphere $(\approx 95 \mathrm{~km})$ at night, to an accuracy of $5 \mathrm{~m} / \mathrm{s}$. The horizontal wind vector is measured by observing the Doppler shift of rotational lines of molecular oxygen aleng two lines of sight. ${ }^{3.4}$ In addition to winds, temperatures and volume emission rates are obtained in the mesosphere and lower thermosphere, and cloud top heights, effective albedos, aerosol phase functions, and scattering coefficients are determined in the stratosphere. Figure 1 schematically depicts the physical processes involved in the measurement. Depending on the band and atmospheric region, molecular-oxygen lines can be observed in either emission or absorption, the latter dominating below $\approx 50 \mathrm{~km}$. Solar radiation incident on the atmosphere is absorbed, exciting molecular oxygen into the $\mathrm{O}_{2}\left(b^{1} \Sigma_{g}^{+}\right)$state. This can occur directly through resonance or photochemical reactions. ${ }^{5.6}$ When this 


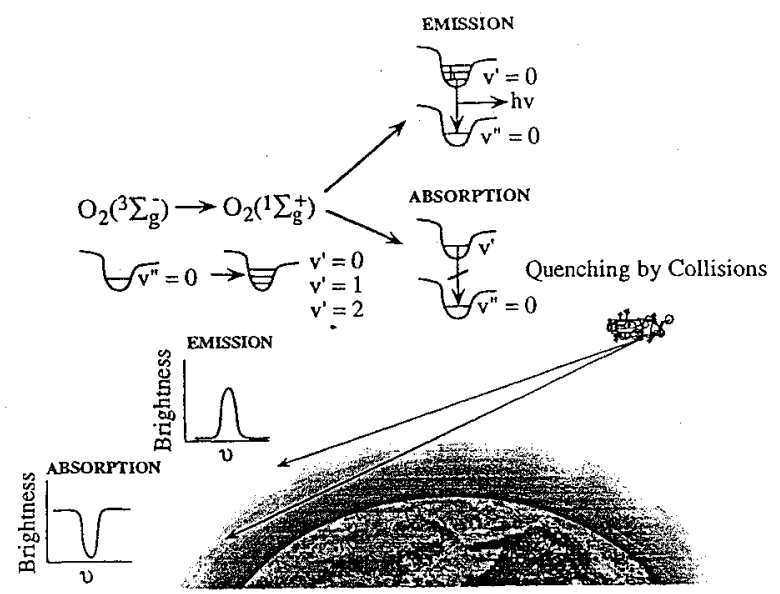

Fig. 1 Schematic illustrating the physical processes involved in the HRDI measurement.

process takes place in the mesosphere or above, where quenching effects are small, the $\mathrm{O}_{2}$ emission lines in the $A$ band of the $\left(b^{1} \Sigma_{g}^{+}, v^{\prime}=0-X^{3} \Sigma_{g}^{-}, v^{\prime \prime}=0\right)$ transition are observable. The emission in the $B(1,0)$ and $\gamma(2,0)$ bands can be ignored because the excited vibrational states are collisionally quenched into the $v^{\prime}=0$ state before the electronic transition takes place. ${ }^{7-9}$ Below $\approx 50 \mathrm{~km}$ electronic quenching $\left(b^{1} \Sigma_{g}^{+}-X^{3} \Sigma_{g}^{-}\right)$rather than emission dominates the loss process. In this case, the absorbed photons are not reemitted, and absorption lines develop in the spectrum. Absorption features in the $\mathrm{A}\left(\approx 762 \mathrm{~nm}, 13,120 \mathrm{~cm}^{-1}\right), \mathrm{B}(\approx 688 \mathrm{~nm}, 14,525$ $\left.\mathrm{cm}^{-1}\right)$, and $\gamma\left(\approx 629 \mathrm{~nm}, 15,902 \mathrm{~cm}^{-1}\right)$ bands can be observed when solar radiation is elastically scattered by molecules and aerosols into the field of view of the observer. This scattering may occur before or after light is reflected from the surface. The line features generated by the atmospheric bands (see Ref. 3 ) are generally quite narrow, with a width of $\sim 0.001 \mathrm{~nm}\left(2 \times 10^{-2} \mathrm{~cm}^{-1}\right)$, and are therefore suitable for the Doppler-shift measurement technique.

The HRDI and early results have been discussed elsewhere. ${ }^{10-16}$ This paper will review the instrument, discuss some of the important in-flight calibrations, and give examples of the type of data recovery possible. Several atmospheric parameters can be obtained with HRDI, but this paper will deal exclusively with the recovery of winds.

\section{Instrumentation}

\section{Theory of Operation}

A typical horizontal wind speed in the middle atmosphere of $10 \mathrm{~m} / \mathrm{s}$ causes a Doppler shift of $\approx 2 \times 10^{-5} \mathrm{~nm}\left(4 \times 10^{-4} \mathrm{~cm}^{-1}\right)$. This small shift requires a high-resolution spectrometer with high sensitivity and very good stability. The HRDI is a multiple-etalon Fabry-Perot interferometer designed to achieve these goals. The Fabry-Perot was chosen because of its large light-gathering power and high resolution. ${ }^{17}$ Since complete descriptions of the FabryPerot interferometer have been given elsewhere, ${ }^{18.19}$ only an outline is provided here. The Fabry-Perot interferometer is a deceptively simple device that consists of two transparent plates that are extremely flat (up to $\lambda / 200$ ) and parallel (tilt $<2 \times 10^{-11} \mathrm{rad}$ ). The inside surfaces are coated with a fairly high-reflectivity coating $(\approx 80$ $\approx 95 \%$ ), which is usually a multilayer dielectric stack. The device is a resonating cavity that forms Haidinger fringes at an infinite distance from the etalon. The fringes are relocated to a finite distance by the use of imaging optics. The transmittance for an ideal Fabry-Perot etalon is given by the Airy function

$$
T(M)=\frac{(1-R)^{2}}{1-2 R \cos (2 \pi M)+R^{2}}
$$

where $M$ in turn is given by

$$
M=2 \mu t \nu \cos \theta
$$

The peak transmittance of such an ideal etalon is unity and occurs whenever the order of interference is an integer. This can occur for

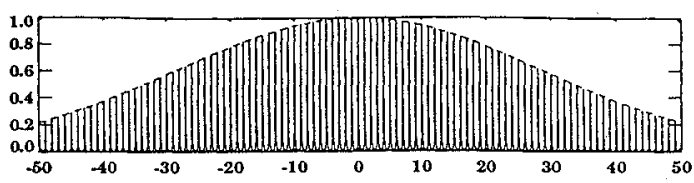

a)

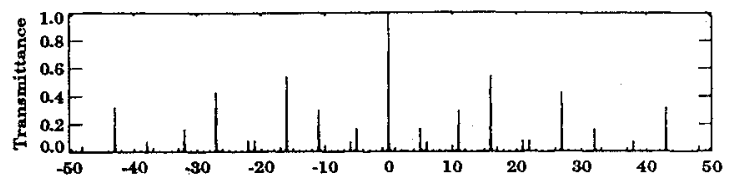

b)

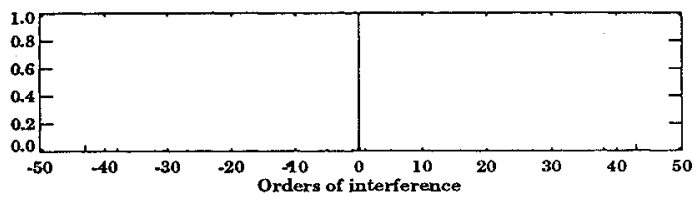

c)

Fig. 2 Illustration of how multiple etalons change the instrument function. a) Single etalon with a 1-cm gap and $0.8-\mathrm{nm}$-wide interference filter. b) Same as a), but with a second etalon with a $0.186-\mathrm{cm}$ gap. c) Same as b), but with a third etalon with a $0.025-\mathrm{cm}$ gap.

an infinity of combinations of $\mu, t, \theta$, and $\nu$. If we vary only the wave number, the separation between peaks at normal incidence is the free spectral range:

$$
\Delta v_{\mathrm{FSR}}=\frac{1}{2 \mu t}
$$

If $M$ is an integer for the conditions $\mu=1$ (vacuum), $t=t_{0}, \theta=$ $\theta_{0}=0$, and $v=v_{0}$, then in order for $M$ to remain an integer the following condition must be satisfied:

$$
\frac{\Delta v}{v}=\frac{\theta^{2}}{2}-\frac{\Delta t}{t_{0}}
$$

Equation (4) illustrates that for space-flight applications there are two practical methods for scanning a Fabry-Perot interferometer in wave number: 1) by looking at different angles through the interferometer, which amounts to viewing different spatial locations at the focal plane of the imaging optics, and 2) by varying the thickness of the etalon gap. Both of these techniques are utilized by the HRDI.

The Airy function describes a periodic response, as shown in Fig. 2a. The instrument response is adequate to observe narrow spectral features with a very small background, so that light will be transmitted through only one order. This requirement is satisfied in the upper atmosphere, where widely spaced emission lines are viewed against a dim background. The line of interest is isolated by adding a low-resolution interference filter. In the lower atmosphere (below the stratopause), the HRDI observes absorption lines in scattered sunlight, and a single etalon would transmit light through all orders, causing the signal to be overwhelmed by the "parasitic"light. It is necessary to use a multiple-etalon system in order to reduce this undesired light, ${ }^{20.21}$ and careful selection of the etalon thicknesses ${ }^{22}$ lowers the parasitic light of the instrument to an acceptable level. The effect of adding a second and third etalon with an interference filter to the system is shown in Figs. $2 b$ and $2 c$. The HRDI employs one etalon with a fixed gap of $1.0 \mathrm{~cm}$, which is scanned by observing different angles through the etalon using a multichannel detector. The gaps of the second and third etalons (0.186- and $0.025-\mathrm{cm}$ thickness) can be varied slightly $(\approx 1000 \mathrm{~nm}$ ) with piezoelectric materials so that they may be adjusted into resonance with any order of the fixed, high-resolution etalon. This allows the instrument to be scanned in wavelength over a range that is limited only by the interference filter. 


\section{Instrument Requirements}

The requirement to measure a Doppler shift on the order of $10^{-4} \mathrm{~cm}^{-1}$ demands an instrument that has high throughput and high spectral resolution. The Fabry-Perot interferometer that is used in the HRDI is such a device (see Ref. 17). Throughput and resolution are not independent parameters, and optimization must be performed to define the best system. The optimization procedure must consider practical limitations. For example, the resolution (FWHH) of a Fabry-Perot is given by

$$
\Delta \nu_{\text {res }}=\frac{\Delta v_{\mathrm{FSR}}}{N}
$$

and $N$ is a combination of the finesses due to reflectivity, instrument defects, and detector broadening. To a good approximation, it is given by

$$
\frac{1}{N^{2}}=\frac{1}{N_{R}^{2}}+\frac{1}{N_{D}^{2}}+\frac{1}{N_{A}^{2}}
$$

The amount of light collected in the central order (assuming no true absorption) is close to

$$
\Delta \bar{\nu}=\frac{\pi \sqrt{R} \Delta \nu_{\mathrm{FSR}}}{(1+R) N_{R}}
$$

There are three important features to note from these equations. First, the amount of light collected decreases monotonically as the reflectivity increases. Second, the light in the central order depends only on the free spectral range (which in turn depends on the gap spacing) and the etalon reflectivity, while the resolution depends on the defects as well. Defects increase the interferometer half-width and decrease the peak transmission. Third, if the finesses are significantly different, then the resolution is determined by the smallest finesse. A practical rule is to try to make the finesses approximately equal. The HRDI detector is a multichannel device which has 32 channels equally spaced in wavelength. The number of channels was limited by telemetry and manufacturing capabilities. The aperture finesse is approximately the number of channels per order. This was set equal to 30 at $762 \mathrm{~nm}$ (i.e., $\approx 1$ order on the detector), and it decreased linearly with decreasing wavelength to 25 at $630 \mathrm{~nm}$. The Fabry-Perot plates were manufactured to a matched flatness of $\approx \lambda / 200$, and in order to insure that the etalons would survive launch stresses, they were firmly attached to their mounts. This had the unfortunate effect of significantly stressing and distorting the plates so that the defect finesses were about 20 . The reflectivity of the etalons was about 0.90 at all wavelengths of interest, corresponding to a reflectivity finesse of about 30 . These combine to give an overall finesse of about 12 . The large degree of distortion imposed by the etalon mountings was unanticipated, and the etalons were coated to a higher than optimal value.

The quantity of light collected by a Fabry-Perot is proportional to the effective area of the etalon and the solid angle passing through it. The solid angle through the etalon is

$$
\Omega_{\mathrm{etl}}=2 \pi \frac{\Delta \nu_{\mathrm{etl}}}{\nu_{0}}
$$

The HRDI examines a spectral range of $0.5 \mathrm{~cm}^{-1}$ at $\approx 13,100 \mathrm{~cm}^{-1}$. The solid angle is then approximately $2.4 \times 10^{-4} \mathrm{sr}$, and, with an effective etalon diameter of $8.9 \mathrm{~cm}$, the area-solid-angle product is $1.49 \times 10^{-2} \mathrm{~cm}^{2}$-sr. This number must be matched by all other optical elements in the system. This requirement is readily met by the rest of the interferometer optics; the only real concern is the telescope that observes the atmosphere. The diameter of the telescope is limited in size by mass constraints, the cost of the primary mirror, and the length of the baffle. A large-diameter telescope requires a long baffle, adding its own mass and moment of inertia, which is a consideration because the telescope must move rather rapidly and with a minimum of wobble. Since the HRDI is a limb viewer, the vertical field of view is limited by the vertical scale of the features in the atmosphere that the instrument is designed to study. The scale height of the atmosphere in the stratosphere, mesosphere, and lower thermosphere ranges from 5 to $8 \mathrm{~km}$. A vertical field of view of $0.12 \mathrm{deg}$ corresponds to a vertical range on the limb of $5 \mathrm{~km}$ at a satellite altitude of $600 \mathrm{~km}$. The horizontal field of view can be much wider, since typical horizontal scales in the atmosphere are much larger than vertical features. Since the HRDI measures the winds by determining the Doppler shift, care must be taken to account properly for the Doppler shift contributed by the spacecraft motion. If the field of view is too large, there will be a significant Doppler-shift variation across the field of view. A consideration of these factors leads to a telescope design that incorporates a primary $17.78 \mathrm{~cm}$ in diameter and field of view of $0.12 \times 1.3 \mathrm{deg}$ (a secondary field of view of $0.12 \times 0.12 \mathrm{deg}$ is available for star observations). The telescope is an off-axis Gregorian, which minimizes small-angle scattering, and the area-solid-angle product is then $1.1 \times 10^{-2} \mathrm{~cm}^{2}$ sr. This demonstrates that the etalon and telescope are not optimally matched. The entire etalon (area and solid angle) is filled by the use of a fiber-optic bundle, which illuminates it with a lower intrinsic brightness than if the etalon and telescope were matched.

\section{Description of Instrument}

The HRDI hardware has been described in some detail elsewhere (see Ref. 12), and only a summary is given here.

The HRDI consists of three components: a telescope for viewing the atmosphere (Fig. 3), an interferometer (Fig. 4), and a microprocessor and electronics. Light from the atmosphere is collected by a fully gimbaled telescope that allows observation on either side of the spacecraft. Light enters the interferometer either from the telescope or from calibration sources (spectral and incandescent lamps), according to the positioning of a scene-selector mirror. The light beam is expanded from $3 \mathrm{~mm}$ in diameter to $25 \mathrm{~mm}$ and passes through two filter wheels. Each filter wheel holds eight filters, and each filter has a bandwidth of $\approx 0.8 \mathrm{~nm}\left(17 \mathrm{~cm}^{-1}\right)$. A small fraction of the light is collected by a photometer, which provides information on the brightness in the broader spectral region. The light beam is then further expanded to $96-\mathrm{mm}$ diameter and passes through the three etalons. The spectrally dispersed beam is focused onto the detector by a telescope with folded optics that allows a $2-m$ focal length to be incorporated in about a $0.5-\mathrm{m}$ space. The detector employs a multichannel concentric-ring design, which spatially scans the highest-resolution etalon in wavelength. This device, known as an image-plane detector (IPD), ${ }^{23-25}$ is a photomultiplier with an anode

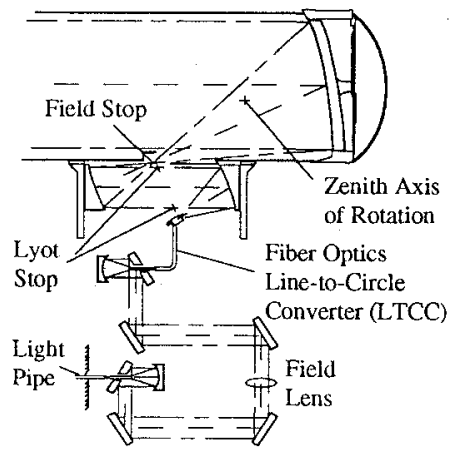

a)

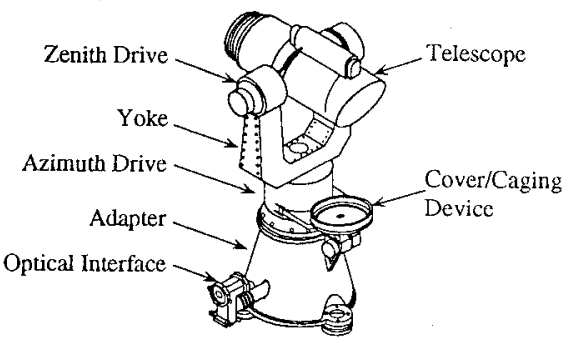

b)

Fig. 3 Drawing of the HRDI telescope. The telescope can rotate around two axes to allow viewing on both sides of the spacecraft and at any altitude in the atmosphere. 

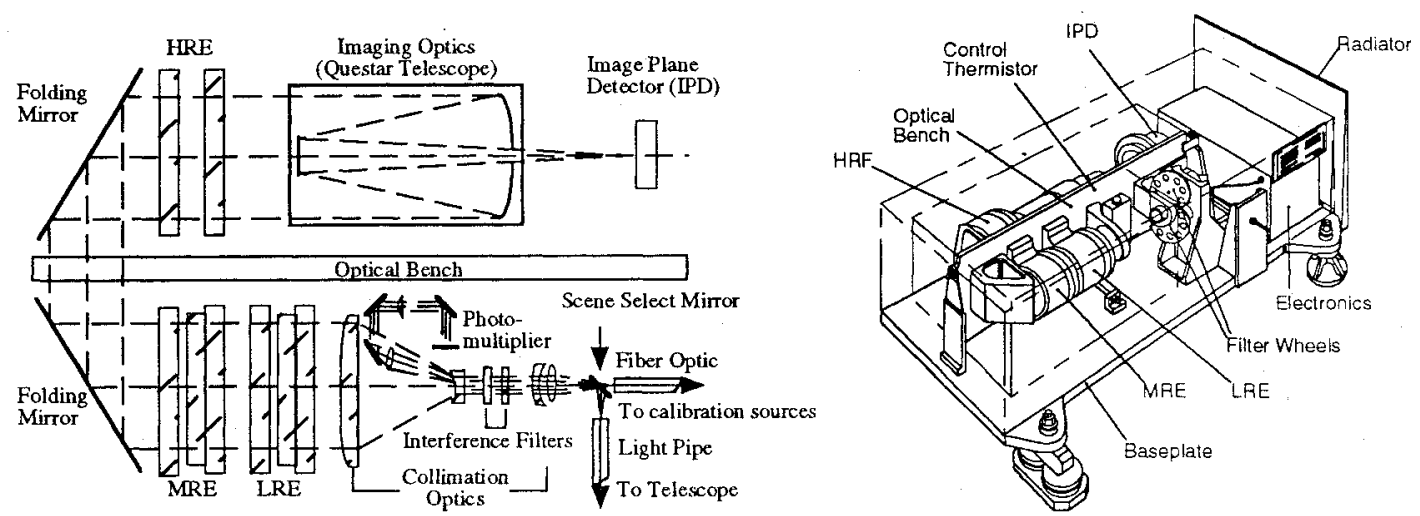

Fig. 4 Optical layout of the HRDI. LRE = low-resolution etalon, MRE $=$ medium-resolution etalon, and HRE $=$ high-resolution etalon.

Table 1 Summary of HRDI parameters

\begin{tabular}{ll}
\hline \hline Telescope & \\
Primary diameter & $17.78 \mathrm{~cm}$ \\
Field of view & $0.12 \times 1.369 \mathrm{deg}$ (wide) \\
& $0.12 \times 0.12 \mathrm{deg}$ (narrow) \\
Transmittance & $\approx 25 \%$ \\
Detector & 32 \\
$\quad$ Number of channels & $0.096 \mathrm{~s}$ \\
Integration period & $0.032 \mathrm{~s}$ \\
Off period & $0.128 \mathrm{~s}$ \\
Repetition period & $0.053(631 \mathrm{~nm})$ \\
Effective quantum efficiency & $0.043(691 \mathrm{~nm})$ \\
& $0.037(725 \mathrm{~nm})$ \\
& $0.030(768 \mathrm{~nm})$ \\
Dark signal & $0.63($ counts $/ 0.096 \mathrm{~s}) / \mathrm{channel}\left(-10^{\circ} \mathrm{C}\right)$ \\
& $1.47(\mathrm{counts} / 0.096 \mathrm{~s}) / \mathrm{channel}\left(-5^{\circ} \mathrm{C}\right)$ \\
Etalons & \\
Plate diameter & $13.2 \mathrm{~cm}$ \\
Coated diameter & $9.4 \mathrm{~cm}$ \\
Plate material & Spectrosil-B \\
Coating materials & ZnS-ThF \\
Post type & Fixed $(\mathrm{HRE})$ \\
Post material & Piezoelectric $(\mathrm{MRE}$ and HRE) \\
& Zerodur $(\mathrm{HRE})$ \\
Interferometer & PZT-5H + quartz $(\mathrm{MRE}$ and LRE) \\
Spectral range & \\
Number of filters & $630-770 \mathrm{~nm}$ \\
Filter bandwidth & 13 \\
Spectral resolution $(\mathrm{HWHH})$ & $\approx 0.020 \mathrm{~cm}-1$ \\
Continuum sensitivity & $\approx 1 \times 10^{-4}$ (counts $\left./ 0.096 \mathrm{~s}\right) /\left(\mathrm{rad} / \mathrm{cm}^{-1}\right)$ \\
\hline- & \\
\hline
\end{tabular}

pattern that duplicates the interference pattern of the interferometer. This allows multiple wavelengths to be sampled simultaneously. For the device used on the HRDI, 31 wavelengths are sampled and the spectral width of each channel is about $0.001 \mathrm{~nm}$. The HRDI is controlled by a dedicated microcomputer, which is necessary in order to adjust the spacing of the etalon gaps, to compensate for thermal drifts, and to point the telescope. Table 1 summarizes the HRDI instrument parameters.

\section{Determination of Wind Shift}

In order to determine winds, which is the primary purpose of the HRDI, it is necessary to determine the Doppler shift of an absorption or emission line. Figure 5a shows the signal observed by an emission line on the IPD with and without a Doppler shift. The unshifted line is centered on channel 16 , and the shifted line is moved by $20 \mathrm{~m} / \mathrm{s}$. Figure $5 \mathrm{~b}$ clearly demonstrates that the shift has a very small effect at line center and in the wings, and is most notable on the sides of the line. The Doppler shift causes the signal in some channels to increase and in others to decrease. The proper interpretation of these signal changes allows the Doppler shift to be determined. The algorithm used to recover the Doppler shift is complex, and only a conceptual demonstration of the technique is presented here. The

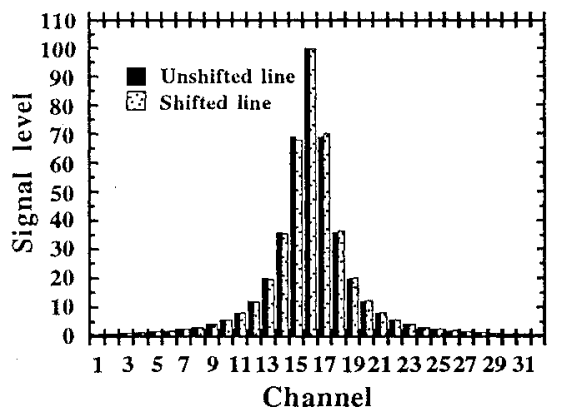

a)

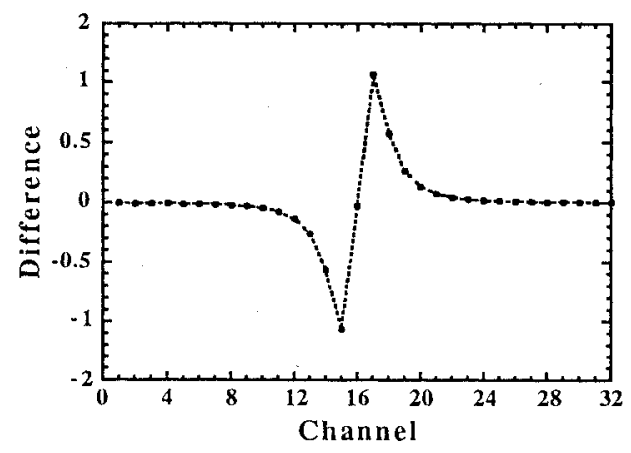

b)

Fig. 5 Illustration of the effect Dopper shifts have on the spectrum observed by HRDI. a) Spectra with and without a small Doppler shift. b) The difference between the two spectra. The center and wings are unaffected, but the sides show a dramatic change.

signal in the presence of a shifted emission or absorption line is given by.

$$
C_{i}(U)=C_{i}(0)+\left.\frac{\partial C}{\partial U}\right|_{i} U
$$

Equation (9) can be inverted in a least-squares fashion, using all channels, and converted by the Doppler equation to give

$$
U=\frac{\left.\frac{c}{v} \sum_{i}\left(\frac{C_{i}(U)-C_{i}(0)}{C_{i}}\right) \frac{\partial C}{\partial v}\right|_{i}}{\sum_{i} \frac{1}{C_{i}}\left(\left.\frac{\partial C}{\partial v}\right|_{i}\right)^{2}}
$$

The statistical uncertainty of velocity can be estimated by assuming the only source of error is photon statistics in $C_{i}(U)$. This is the largest source of error and can adequately describe the statistical error. Using Poisson statistics which describe photon noise, the 
uncertainty in $U$ is given by

$$
\begin{aligned}
& \sqrt{\delta^{2} U}=\frac{c}{v} \frac{1}{\left[\sum C_{i}\left(\left.\frac{\partial C}{\partial v}\right|_{i}\right)^{2}\right]^{\frac{1}{2}}} \\
& =\frac{c}{v} \frac{1}{\sqrt{C_{\max }}} \frac{1}{\left[\sum \frac{C_{i}}{C_{\max }}\left(\left.\frac{1}{C_{i}} \frac{\partial C}{\partial v}\right|_{i}\right)^{2}\right]^{\frac{1}{2}}}
\end{aligned}
$$

Equation (11) demonstrates that to minimize wind errors the signal should be as bright as possible and the slope of the signal should be large. The instrumental width $\left(\approx 0.001 \mathrm{~nm}, 0.02 \mathrm{~cm}^{-1}\right)$ is significantly greater than the shift due to atmospheric winds. It is important to recognize that the HRDI measures Doppler shifts on the order of $10^{-5} \mathrm{~nm}$, and is not attempting to resolve spectral features of this magnitude. Since the width of the spectral line depends on convolution of the instrument width and the atmospheric line shape, there is no reason to make the instrument resolution much narrower than the line, as the resultant signal will not thereby become sharper and the signal-to-noise ratio will decrease.

\section{Mode of Operation}

The design of the HRDI allows for versatile programming of operational modes, which are stored in the computer's memory as lookup tables. These tables contain information on the telescope pointing, interferometer tuning wavelength, and dwell time at each altitude. A single mode is described to illustrate the procedure. The example employed for this purpose is the stratospheric daytime wind mode, which scans the stratosphere from 10 to $40 \mathrm{~km}$ with a horizontal resolution of $\approx 500 \mathrm{~km}$. The telescope initially looks forward, at an azimuth of about $45 \mathrm{deg}$ to the spacecraft velocity vector, and vertically scans the atmosphere in $2.5-\mathrm{km}$ steps, pausing at each tangent altitude long enough to collect a sufficient signal to meet the required statistical accuracy (see Fig. 6). The telescope scans from the lowest altitude to the highest in the $B$ band and repeats the altitude grid, using the $\gamma$ band as it scans downward again. After this sequence is performed, the telescope slews to look backwards, at an azimuth of $135 \mathrm{deg}$ to the velocity vector. The time for the two vertical scans and azimuthal slew is about one minute. The telescope then repeats the two vertical scans and slews back to the forward-looking direction. This cycle continues until the computer issues the commands to begin a new mode. For the stratospheric daytime wind mode, a backward-viewing scan will sample very nearly the same volume in space as a forward-viewing scan performed 9 min earlier. The look directions at this volume will differ by $90 \mathrm{deg}$, allowing the two measurements to be combined to give the horizontal wind vector.

The data from the HRDI consist of spectrograms of 32 points ( 31 from the interferometer and one from the photometer channel), the telescope pointing direction, and a number of parameters describing the thermal and electrical state of the instrument. All spectrograms from a given altitude are averaged and corrected for instrumental effects. The line-of-sight Doppler shift is then determined and converted to a line-of-sight wind speed. Since this is not a point measurement but represents the integral effect of different atmospheric

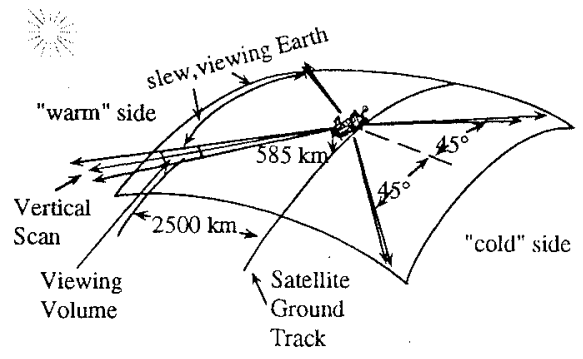

Fig. 6 Illustration of the telescope viewing during a normal science mode. Actual modes will look on just one side of the spacecraft or the other. Typical HRDI operations look on the warm side (towards the sun) to maximize the signal and coverage.

\begin{tabular}{|c|c|c|}
\hline Segment & Lamp & Purpose of test \\
\hline All & None & Detector dark count \\
\hline 1 & Incandescent & Interferometer sensitivity \\
\hline 2 & Neon & $\begin{array}{l}\text { Instrument drift in the } 14,427-\mathrm{cm}^{-1} \text { ( } \mathrm{B} \text { band) } \\
\text { region }\end{array}$ \\
\hline 3 & HAK $^{\text {a }}$ & $\begin{array}{l}\text { Instrument drift in the } 13,151-\mathrm{cm}^{-1} \text { (A band) } \\
\text { region }\end{array}$ \\
\hline 4 & Neon & $\begin{array}{l}\text { Instrument drift in the } 15,856-\mathrm{cm}^{-1} \text { ( } \gamma \text { band) } \\
\text { region }\end{array}$ \\
\hline 5 & Neon & MRE, LRE finesses, HRE \& lamp width \\
\hline 6 & None & PLT postresponse test \\
\hline 7 & None & MRE electronics test \\
\hline
\end{tabular}

Table 2 Frequent HRDI calibrations

a Helium-argon-krypton gas mixture.

motions as the line of sight passes through several altitudes, data are then inverted using weighting functions developed by Hays and Abreu. ${ }^{26}$ This yields the horizontal component of the wind velocity along a particular look direction. These are combined with measurements from an orthogonal look direction to provide the vector wind at a given point. The result is an altitude stack of wind vectors along the satellite track. The final step in the processing is to interpolate the data onto a common UARS spatial and temporal grid for use by the scientific community. Data from other sources (rawinsondes, rockets, etc.) are periodically used to verify the velocity recovery and provide the zero velocity reference. ${ }^{27.28}$

\section{In-Flight Performance}

Proper interpretation of the spectra collected by the HRDI requires knowledge of the state of the instrument. A long preflight calibration sequence provided the instrument state before launch. A simplified series of tests are run in orbit to track the long-term performance. This section describes some of these tests and presents the results of monitoring the instrument for more than two years in orbit.

In-flight testing of the instrument includes routine calibrations, which use the four calibration lamps. Two of the lamps are incandesent and provide a continuum source, and two are spectral lamps, one of which contains neon gas, and the other a mixture of helium, argon, and krypton gas. From Fig. 4 it is seen that the calibration sources provide input to the interferometer but give no information on the transmittance of the telescope. Once the transmittance of the interferometer is determined, the signal from the atmosphere must be used to estimate any changes in telescope performance. Table 2 lists the routine calibrations.

It was decided to perform a set of short calibrations of the instrument every day, and to execute them in small segments not longer than 5 to $10 \mathrm{~min}$ in duration. Calibrations are performed every fourth time the satellite crosses the terminator from day to night. Terminator crossings were chosen because the scientific data collected in regions where the solar zenith angle is near 90 deg are very difficult to interpret and therefore of limited value.

In addition to the daily calibrations, other periodic tests and special calibrations are performed. A more extensive version of the daily calibrations is executed once a month on the day that the spacecraft yaws around. Approximately once a week a series of stars are viewed to determine the misalignment between the HRDI telescope reference frame and the UARS attitude system. This calibration is discussed in the next section.

\section{Calibration and Validation}

The line-of-sight (los) wind velocity is derived from the measured centroid position, $v$, of an absorption or emission line on the detector; it is given by

$$
\begin{aligned}
& U_{\text {los }}=U-U_{0}(\nu, \phi)-\alpha\left(T-T_{0}\right)-f(t)-U_{s} \cos \varepsilon \cos \beta \\
& -\frac{2 \pi R_{E}}{T_{E}} \sin \psi \cos \phi
\end{aligned}
$$

where all of the terms have velocity units. The principal task of the validation procedure is to determine the last five terms on the righthand side of Eq. (12). The terms $\alpha$ and $f(t)$ can be obtained from 
in-flight calibration data. In addition, an accurate knowledge of the component of the spacecraft velocity along the look direction and the Earth rotation in the viewing direction is essential. The spacecraft velocity is about $7500 \mathrm{~m} / \mathrm{s}$, so even a small uncompensated component will seriously affect the wind determination. Because of the large Doppler shift induced by the spacecraft, which has the effect of moving the spectrum around on the detector, and because of slight nonuniformities in the detector response, it was not practical to attempt to locate the zero position during prelaunch calibration. Instead, it is determined using atmospheric wind data collected in orbit, in conjunction with dynamical assumptions and correlative measurements made by rockets, balloons, radars, and lidars. Each of the validation requirements listed above are discussed in more detail in the following sections.

The instrument drift (both thermal and temporal) is monitored using observations of spectral lines of on-board calibration lamps. The thermal drift is driven by spacecraft heating cycles, which occur on two time scales. The first of these is about 1 orbital period and arises from the day-to-night solar heating variation experienced on each orbit. This short-term temperature fluctuation amounts to $\approx \pm 0.1 \mathrm{~K}$ and causes less than a $1-\mathrm{m} / \mathrm{s}$ drift in the instrument (see Fig. 7). The second temperature variation, which has a magnitude of about $\pm 1 \mathrm{~K}$, occurs with a period of approximately one month. This effect is due to the precession change in the angle between the direction of the sun and the plane of the satellite orbit, called the solar beta

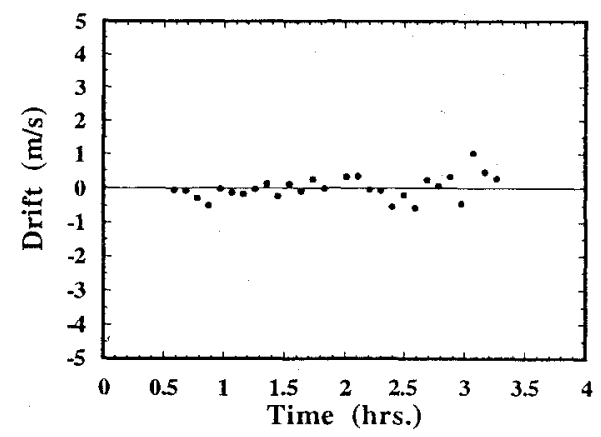

Fig. 7 The stability of the HRDI instrument for a short time period, illustrating the lack of sensitivity to orbital variations. angle. Longer term drifts in the zero reference position are determined by analysis of daily calibration data. Figure $8 \mathrm{a}$ indicates the variation in the position of the $14,427.1443-\mathrm{cm}^{-1}(692.94673-\mathrm{nm})$ neon calibration line on the HRDI detector as observed since the beginning of the mission. In Fig. $8 \mathrm{~b}$ the data have been corrected for the temperature sensitivity of the instrument, leaving a residual long-term drift. The large change during the first 100 days is probably due to exponentially decreasing effects such as outgassing, as the instrument gradually adjusted to the space environment. The break in calibration results from day 201 to 226 arose from a temporary malfunction in the HRDI. The data gap and associated shift in line position between days 265 and 310 corresponds to a period during which the UARS solar array was not operating properly, and consequently spacecraft power was mainly restricted to essential systems. While the precise cause of the resultant line shift is still under investigation, it is suspected to have arisen as a result of the extreme low temperatures (approximately $-20^{\circ} \mathrm{C}$ ) experienced by the HRDI during the extended intervals when instrument power was switched off. In any case, the data may be readily corrected for the observed long-term drift (Fig. 8c). The effect of temperature variations and the long-term drift are continuously re-evaluated for all of the lines employed by the instrument, and the appropriate corrections, accurate to within a few meters per second, are applied to the HRDI atmospheric observations.

The spacecraft velocity of $7500 \mathrm{~m}-\mathrm{s}^{-1}$ is large compared to the typical winds in the atmosphere, and so steps must be taken to remove the associated Doppler shift from the measured detector signal. The uncertainty in the required correction lies not in the accuracy of the satellite velocity, which is known to better than $1 \mathrm{~m} / \mathrm{s}$, but in the location of the telescope viewing direction relative to the satellite trajectory. The spacecraft bus attitude is known to about $0.01 \mathrm{deg}$, which translates to a wind measurement error of only $\approx 1 \mathrm{~m} / \mathrm{s}$. However, the relationship between the spacecraft reference frame and the telescope frame is uncertain. This relationship was determined in preflight calibrations, but is subject to change due to launch shifts, relocation in a zero-gravity environment, and differential heating of the spacecraft during the solar beta cycle. The first two of these effects should not have changed since orbit was achieved, but the beta-cycle variation is time-dependent. The misalignment between the spacecraft frame and the HRDI instrument frame is measured periodically by observing the passage of a series

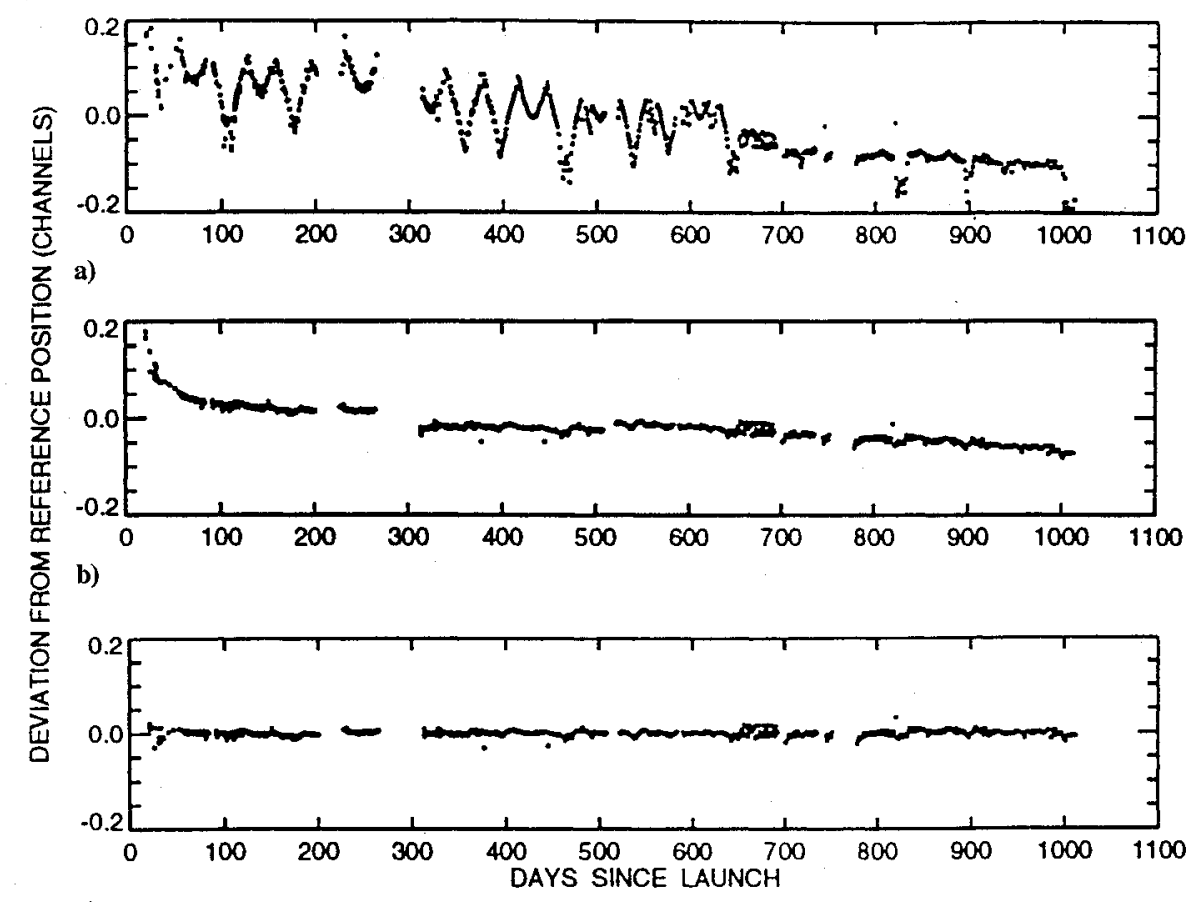

c)

Fig. 8 Position on the HRDI detector in velocity units of the neon calibration line at $14,427.1443 \mathrm{~cm}^{-1}(692.94673 \mathrm{~nm})$. a) Observed location of the calibration line. b) Position of the calibration line after correction for the temperature sensitivity of the instrument, leaving a residual long-term drift. c) Position of the calibration line corrected for temperature effects and the long-term drift. 


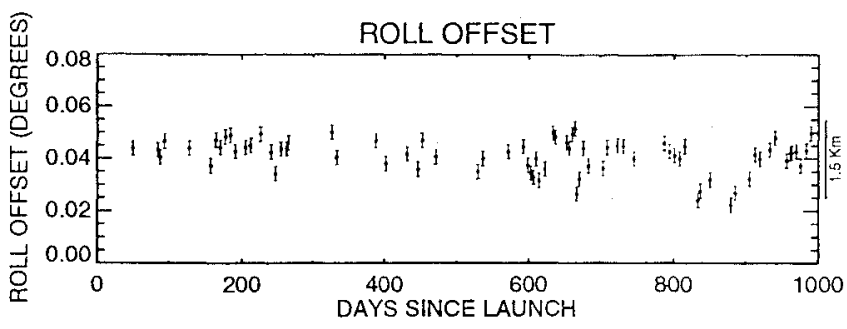

a)

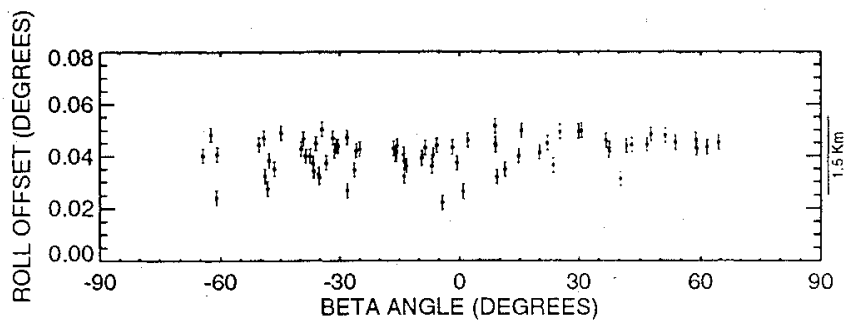

b)

Fig. 9 Determination of the misalignment in the roll axis between the HRDI telescope and the spacecraft coordinate system. The bias in all axes indicates either a launch shift or the effect of changing from a $1 \mathrm{~g}$ environment to $0 \mathrm{~g}$.

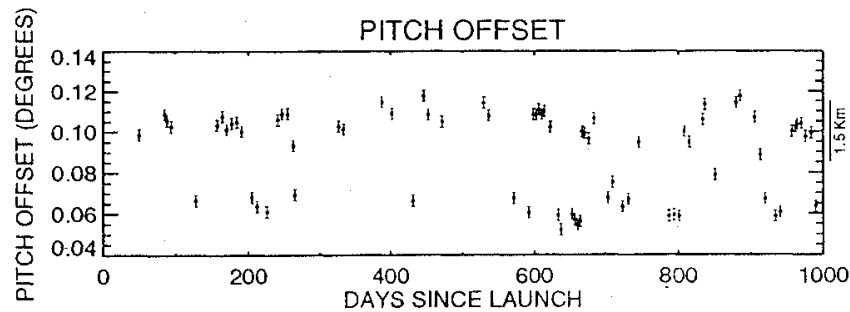

a)

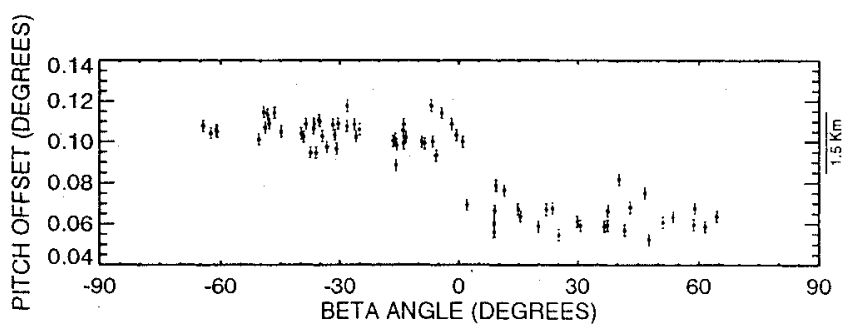

b)

Fig. 10 Determination of the misalignment in the pitch axis between the HRDI telescope and the spacecraft coordinate system. The bias in all axes indicates either a launch shift or the effect of changing from a $1 \mathrm{~g}$ environment to $0 \mathrm{~g}$.

of stars through the telescope field of view. By determining the difference between the measured crossing time of the star through the field of view and a calculated crossing time based on knowledge of the spacecraft ephemeris and the star's position in the sky, it is possible to derive the roll, pitch, and yaw misalignments. Misalignment measurements are performed weekly, and the corresponding corrections are incorporated into the wind analysis algorithms. Figures 9, 10 , and 11 show the determinations of the misalignments between the HRDI and spacecraft frames for roll, pitch, and yaw. The pitch misalignment in particular exhibits a strong beta-angle dependence, and varies over a range of $0.05 \mathrm{deg}$, corresponding to an uncertainty in the wind determination on the order of $5 \mathrm{~m} / \mathrm{s}$. This result was somewhat surprising at first, but we theorize that the alignment difference is due to the fact that the spacecraft itself is yawed around $180 \mathrm{deg}$ when the beta angle changes sign, to keep the sun on the same side of the spacecraft (toward the solar panel and away from the instruments' radiators). These two states of the spacecraft-sun alignment may cause differences in the way the spacecraft structure

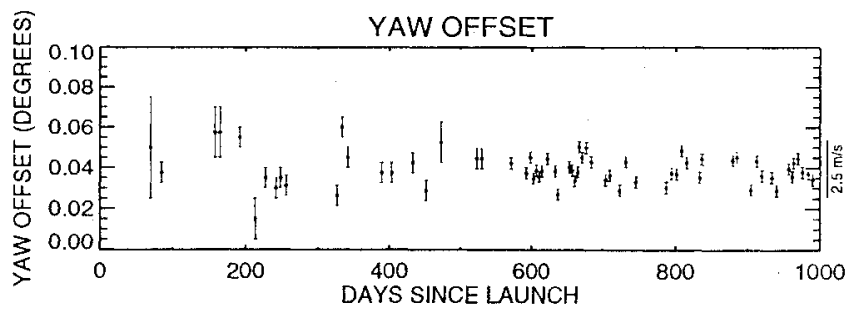

a)

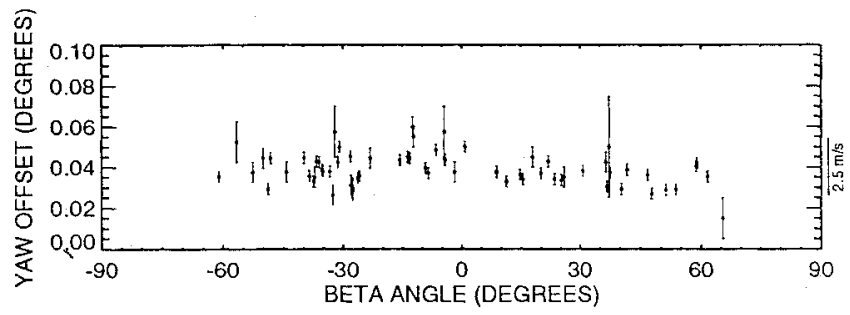

b)

Fig. 11 Determination of the misalignment in the yaw axis between the HRDI telescope and the spacecraft coordinate system. The bias in all axes indicates either a launch shift or the effect of changing from a $1 \mathrm{~g}$ environment to $0 \mathrm{~g}$.
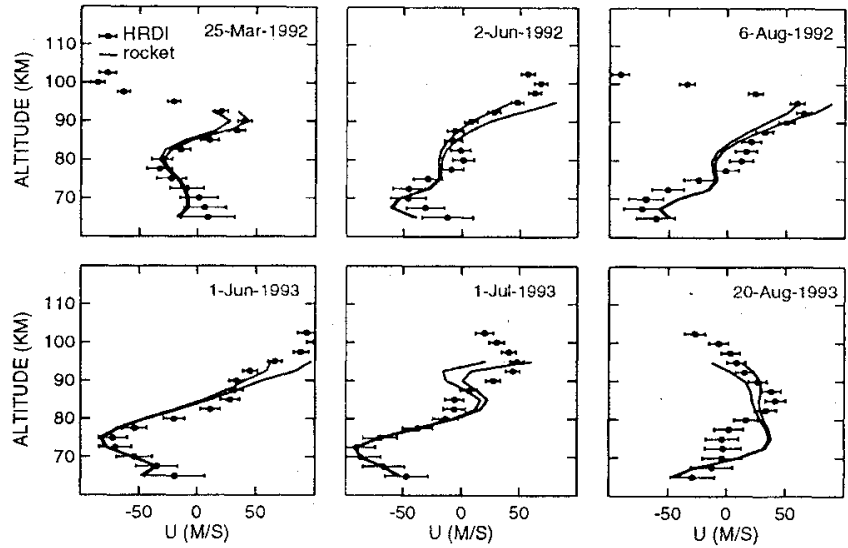

Fig. 12 Comparisons of zonal wind profiles measured by the HRDI (error-barred points) and six rockets (solid lines) launched from Wallops Island.

is heated by the sun, and thus change the misalignment between the spacecraft and the HRDI.

The in-flight calibration data provides information on changes in the zero reference position, but not its absolute value. This parameter is determined from geophysical constraints and correlative data. Two examples of reasonable geophysical constraints for the mesosphere are that the global mean winds sampled at the same local time do not change rapidly over a period of a day or two and that the long-term global mean meridional winds are zero. The two constraints have yielded estimates of the reference positions accurate to within about $10 \mathrm{~m} / \mathrm{s}$.

Further improvement in the accuracy of the zero reference positions requires the comparison of HRDI measurements with other UARS instruments, particularly the wind imaging interferometer (WINDII), ${ }^{29}$ ground-based instrumentation (lidars, radars, etc.), special in situ measurements (balloons, rockets), and the results of models and climatology. Examples of comparisons between winds observed by the HRDI with those obtained by rockets launched from Wallops Island are presented in Fig. 12. It is a nontrivial problem to compare satellite remote sensing observations with other types of data. Correlative instruments typically provide vertical profiles more or less directly above the station, yielding essentially point measurements. In contrast, limb-viewing satellite instruments such as the HRDI give profiles that are a weighted average along the line of sight. There are also uncertainties associated with the averaging procedures employed by the correlative measurement techniques. 


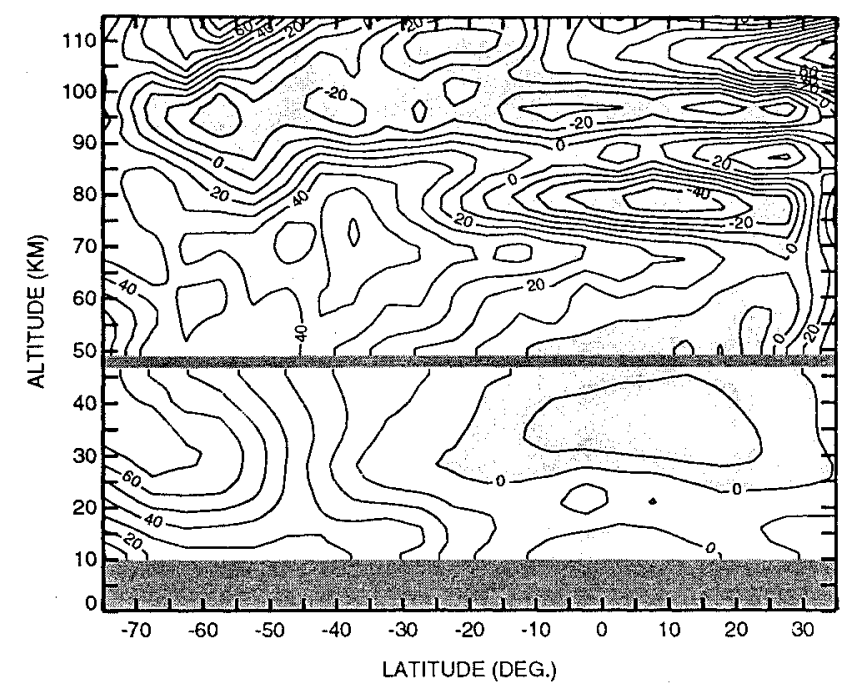

Fig. 13 Example of HRDI-measured winds. This shows the zonal winds in the stratosphere, mesosphere, and lower thermosphere averaged from August 14 to September 16, 1993.

Because of the different effective spatial and temporal resolutions of HRDI and correlative measurements and the high degree of shortterm atmospheric variability, this means that the various techniques are not really sampling the same quantity. In addition, most inversion methods (including those used by the HRDI) employ filtering techniques designed to remove altitude oscillations that are purely artifacts of the analysis procedure. These may have varying degrees of success, and it is possible that real variations with various size scales will be removed from the data. Another difficulty is that the coincidence of the HRDI and correlative measurements is never exact, either in space or time. Because of the problems outlined above, the comparison of HRDI data with other measurements on a profile-by-profile basis is of limited value. It is necessary instead to view the data sets from a statistical perspective. One approach is to determine the mean differences between the HRDI and correlative measurements for each site using as many coincidences as possible (typically $10-50$ ). This information allows the zero velocity to be determined to within a few meters per second (see Refs. 27 and 28).

\section{Conclusions}

A high-resolution, high-throughput, and extremely stable FabryPerot interferometer has been constructed for the direct measurement of Doppler shifts of atmospheric absorption and emission lines. Observations of the winds in the middle atmosphere have been made since October 1991. An example of the data collected by the HRDI is given in Fig. 13, which shows the zonal winds in the stratosphere, mesosphere, and lower thermosphere. The wind field is presented as a function of latitude and altitude for a local solar time of 16:00, and data were collected from August 14 to September 16, 1993. This corresponds to late winter in the southern hemisphere, and the signature of the Antarctic stratospheric vortex is clearly visible. In the mesosphere, the winds in the south are characterized by winter westerlies. The oscillatory behavior with altitude of the winds in the low-latitude mesosphere and lower thermosphere regions is due to the $(1,1)$ diurnal tide.

\section{Acknowledgment}

This work was sponsored by NASA through Contract NAS527751 .

\section{References}

${ }^{1}$ Andrews, D. J., Holton, J. R., and Leovy, C. B., Middle Atmosphere Dynamics, 1st ed., Academic, New York, 1987.

${ }^{2}$ Reber, C. A., "The Upper Atmosphere Research Satellite," EOS, Vol. 71, No. 51, 1990, pp. 1867-1878.

${ }^{3}$ Hays, P. B., "High-Resolution Optical Measurements of Atmospheric Winds from Space. 1: Lower Atmosphere Molecular Absorption," Applied Optics, Vol. 21, No. 6, 1982, pp. 1136-1141.

${ }^{4}$ Abreu, V. J., Bucholtz, A., Hays, P. B., Ortland, D. A., Skinner, W. R., and
Yee, J.-H., "Absorption and Emission Line Shapes in the $\mathrm{O}_{2}$ Atmospheric Bands: Theoretical Model and Limb Viewing Simulations," Applied Optics, Vol. 28, No. 11, 1989, pp. 2128-2137.

${ }^{5}$ Wallace, L., and Hunten, D. M., "Dayglow of the Oxygen A Band", Journal of Geophysical Research, Vol. 73, No. 15, 1968, pp. 4813-4834.

${ }^{6}$ Bucholtz, A., Skinner, W. R., Abreu, V. J., and Hays, P. B., "The Dayglow of the $\mathrm{O}_{2}$ Atmospheric Band System," Planetary and Space Sciences, Vol. 34, No. 11, 1986, pp. 1031-1035.

${ }^{7}$ Lee, L. C., and Slanger, T. G., "Observations on $O\left({ }^{1} D \rightarrow{ }^{3} P\right)$ and $\mathrm{O}_{2}\left(b^{l} \Sigma_{g}^{+} \rightarrow X^{3} \Sigma_{g}^{-}\right)$Following $\mathrm{O}_{2}$ Photodissociation," Journal of Chemical Physics, Vol. 69, No. 9, 1978, pp. 4053-4060.

${ }^{8}$ Gauthier, M. J., and Snelling, D. R., "Production de $\mathrm{O}_{2}\left(b^{1} \Sigma_{g}^{+}\right), v^{\prime}=$ 0 , 1, et 2 par la reaction $\mathrm{O}\left(2^{1} D_{2}\right)+\mathrm{O}_{2}\left(X^{3} \Sigma_{g}^{-}\right)$," Canadian Journal of Chemistry, Vol. 52, No. 25, 1974, pp. $4007-4015$.

${ }^{9}$ Schurath, U., "The Energy Pooling Reaction $2 \mathrm{O}_{2}\left({ }^{1} \Delta_{g}\right) \rightarrow \mathrm{O}_{2}\left(X^{3} \Sigma_{g}^{-}\right)$ $+\mathrm{O}_{2}\left(b^{1} \Sigma_{g}^{+}\right)$Formation, Relaxation, and Quenching of Vibrationally Excited $\mathrm{O}_{2}\left(b^{l} \Sigma_{g}^{+}\right)$," Journal of Photochemistry, Vol. 4, No. 3, 1975, pp. 215226.

${ }^{10}$ Hays, P. B., Skinner, W. R., Abreu, V. J., and Yee, J.-H., "The High Resolution Doppler Imager," Digest of the Topical Meeting on Optical Remote Sensing of the Atmosphere, Vol. 4, Optical Society of America, Washington, DC, 1990, pp. 7-10.

${ }^{11}$ Abreu, V. J., Hays, P. B., and Skinner, W. R., "The High Resolution Doppler Imager," Optics \& Photonics News, Vol. 2, No. 10, 1991, pp. 2830 .

${ }^{12}$ Hays, P. B., Abren, V. J., Dobbs, M. E., Gell, D. A., Grassl, H. J., and Skinner, W. R., "The High Resolution Doppler Imager on the Upper Atmosphere Research Satellite," Journal of Geophysical Research-Atmosphere, Vol. 98, No. D6, 1993, pp. 10,713-10,723.

${ }^{13}$ Hays, P. B., et al., "Remote Sensing of Mesospheric Winds with the High Resolution Doppler Imager,' Planetary and Space Sciences, Vol. 40, No. 12, 1993, pp. 1599-1606.

${ }^{14}$ Skinner, W. R., Hays, P. B., and Abreu, V. J., "Wind Measurements with a High Resolution Doppler Imager (HRDI)," Proceedings of the NASA Symposium on Global Wind Measurements, A. Deepak, Hampton, VA, 1986 pp. $129-132$.

${ }^{15}$ Skinner, W. R., Hays, P. B., and Abreu, V. J., "High Resolution Doppler Imager," IGARSS'87, 1987.

${ }^{16}$ Morton, Y. T., et al., "Global Mesospheric Tidal Winds Observed by the High Resolution Doppler Imager on Board the Upper Atmosphere Research Satellite," Geophysical Research Letters, Vol. 20, No. 12, 1993, pp. 1263 1266.

${ }^{17}$ Jacquinot, P., "The Luminosity of Spectrometers with Prism, Gratings, or Fabry-Perot Etalons," Journal of the Optical Society of America, Vol. 44, No. 10, 1954, pp. 761-765.

${ }^{18}$ Hernandez, G., Fabry-Perot Interferometers, 1st ed., Cambridge Univ. Press, Cambridge, England, UK, 1986.

${ }^{19}$ Vaughn, J. M., The Fabry-Perot Interferometer, 1st ed., Adam Hilger, Bristol, 1989.

${ }^{20}$ Mack, J. E., McNutt, D. P., Roesler, F. L., and Chabbal, R., "The PEPSIOS Purely Interferometric High-Resolution Scanning Spectrometer. 1. The Pilot Model," Applied Optics, Vol. 2, No. 9, 1963, pp. 873-885.

${ }^{21} \mathrm{McNutt}$, D. P., "PEPSIOS Purely Interferometric High-Resolution Scanning Spectrometer. II. Theory of Spacer Ratios," Journal of the Optical Society of America, Vol. 55, No. 3, 1965, pp. 288-292.

${ }^{22}$ Skinner, W. R., Hays, P. B., and Abren, V. J., "Optimization of a Triple Etalon Interferometer," Applied Optics, Vol. 26, No. 14, 1987, pp. 2817 2827.

${ }^{23}$ Hays, P. B., Killeen, T. L., and Kennedy, B. C., "The Fabry-Perot Interferometer on Dynamics Explorer," Space Science Instrumentation, Vol 5, No. 4, 1981, pp. 395-416.

${ }^{24}$ Killeen, T. L., Kennedy, B. C., Hays, P. B., Symanow, D. A., and Ceckowski, D. H., "Image plane detector for the Dynamics Explorer FabryPerot interferometer," Applied Optics, Vol. 22, No. 22, Nov. 15, 1983, pp 3503-3513.

${ }^{25}$ Hays, P. B., and Wang, W., "Image plane detector for Fabry-Perot interferometers: Physical model and improvement with anticoincidence detection," Applied Optics, Vol. 30, No. 22, Aug. 1, 1991, pp. 3100-3107.

${ }^{26}$ Hays, P. B., and Abreu, V. J., "Absorption Line Profiles in a Moving Atmosphere: A Single Scattering Linear Perturbation Theory," Journal of Geophysical Research, Vol. 94, No. D15, 1989, pp. 18, 351-18,365.

${ }^{27}$ Burrage, M. D., et al., "Comparison of HRDI Wind Measurements with Radar and Rocket Observations," Geophysical Research Letters, Vol. 20, No. 12, 1993, pp. 1259-1262.

${ }^{28}$ Burrage, M. D., et al., "Validation of Winds from the High Resolution Doppler Imager on UARS," in Optical Spectroscopic Techniques and Instrumentation for Atmospheric and Space Research, edited by J. Wang and P. Hays, Proc. SPIE, Vol. 2266, pp. 294-306, 1994.

${ }^{29}$ Shepherd, G. G., et al., "WINDII, the Wind Imaging Interferometer on the Upper Atmosphere Research Satellite," Journal of Geophysical Research, Vol. 98, No. D6, 1993, pp. 10,725-10,750. 